\title{
Radar Recognition through Statistical Classification of Cellular Emission in the Moment Space
}

\author{
Nelson Chávez and Angel L. González \\ Instituto Técnico Militar "José Martî" \\ itm@unicom.co.cu
}

\begin{abstract}
A method is presented for radar target rescognition, using the moments of the parameters of the scattered or emitted signals for classification. Large size samples of signal parameters, formed by signals emitted by each of the resolution cells of the searching region, are used to obtain a determined number of normal distributed moments which represent statistical features of the cellular emissions. Taking these moments as components a classification vector is obtained in the moment space. Classification process is carried out assigning every cell of the searching region to one of the previously determined classes during an adaptive process, where the border limiting each class is determined accordingly Neyman - Pearson criterion. The larger size samples, the lower the classification error, which makes possible the distinction of processes very similarly to each other.
\end{abstract}

Keywords: Target recognition, classification vector, moment space, searching region, distributed targets.

\section{Introduction}

Radar Detection by Analysis and Statistical Classification of Cellular Emission (RADASCE) [ 1 - 5 ], is an adaptive - parametric detection method, which makes possible the achievement of high probabilities of detection particularly, in those cases when the scattering properties of electromagnetic signals of a specific target and its environment are very similar to each other. In this method, the random variables used in the decision process are a number of selected moments of the signal parameters. The essence of this method is presented synthetically as follows.

The surveillance region, scanned by de the radar beam, is divided into resolution cells, see Fig. 1, and a searching gate, of appropriate dimensions, is defined to process the electromagnetic emissions coming from it. The media inside this searching gate is considered to be homogeneous and its associated random processes (parameters of the scattered or emitted signals) stationary in time and space. Changes in physical properties of the media inside a cell will cause a perturbation of the considered random processes. In the case of radar detection (classification between two classes) the affected cellular emissions will be called anomalies, while the rest of the cells, are called the background. Proposed classification method [ 2 ] is based on the statistical properties of parameters of emitted or scattered signals by resolution cells. These 

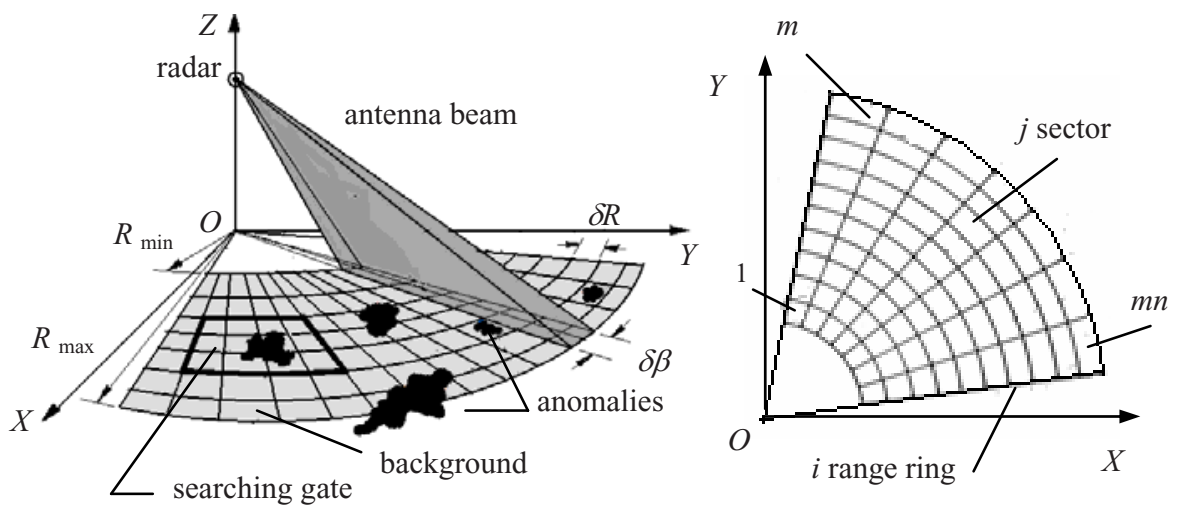

Fig. 1. Division of the searching region into resolution cells

random processes are represented by $\xi_{u}^{w}(t)$, where $u=1, \ldots, m n$, is the resolution cell order and $w=1, \ldots, W$ - the selected signal parameter, e.g., amplitude, phase, polarization angle, etc. The process $\xi_{u}^{w}(t)$ can be represented by a vector, whose components are the selected moments obtained from the samples of the chosen signal parameters. An adequate selection of moments makes possible the clustering of the random processes of the resolution cells into classes. When the sample size $M$ of a signal parameter reaches large values, theoretically when $M \rightarrow \infty$, the distributions of its moments tend to the normal; the detection method becomes parametric and the probability of detection tends to one [ 1 ]. These two facts give the method the posibilities of detection of very low radar cross section targets immerse in clutter and the recognition of processes similar to each other that occur in distributed targets.

\subsection{Classification Space}

From each of the $M$ size samples of the random process $\xi_{u}^{w}(t)$, are obtained moments $m_{k_{1}, \ldots, k_{g}}\left(t_{1}, \ldots, t_{g}\right)$, where $g=1, \ldots, G$, is the order of the statistic being used in the process, and $\sum_{s=1}^{g} k_{s}$ the order of the moment [ 6 ]. A determined number of moments $m_{q}(u, w, g)$, properly selected, where $q=1, \ldots, Q(w, g)$ is the order assigned them, is assigned to each one of the parameter-statistic pairs. To each cellular emission will corresponds a classification matrix $\mathcal{M}_{c}(u)$, of $W$ rows and $G$ columns [2 - 4], given by

$$
\mathcal{M}_{\mathbf{c}}(u)=\left\|\varsigma_{w, g} I_{w, g}(u)\right\|, \varsigma_{w, g}=\left\{\begin{array}{l}
1, \text { if statistic of order } g \text { exists } \\
0, \text { if statistic of order } g \text { doesn't exists }
\end{array} .\right.
$$

where,

$$
I_{w, g}(u)=\left\lfloor m_{1}(u, w, g), \ldots, m_{Q(w, g)}(u, w, g)\right\rfloor .
$$


The classification vector $I(u)$, in an $\boldsymbol{n}$ dimensional space is given by

$$
\begin{gathered}
I(u)=\sum_{w=1}^{W} \sum_{g=1}^{G} \varsigma_{w, g} I_{w, g}(u) . \\
\varkappa=\sum_{w=1}^{W} \sum_{g=1}^{G} Q(w, g) .
\end{gathered}
$$

To every cell of the observation space will correspond one and only one vector in the classification space. On the contrary, a vector in the classification space could be associated to more than one cell in the observation space.

\subsection{Contrast Ratio between Classes: Anomaly / Background Ratio}

Let's suppose $m_{q}^{C_{1}}(w, g), m_{q}^{C_{1}, P}(w, g)$, are the sample and population moments, respectively, corresponding to class $C_{1}$, and $m_{q}^{C_{2}}(w, g), m_{q}^{C_{2}}{ }^{2} P(w, g)$, those to class $C_{2}$. By definition [ 2 ], sample contrast ratio of class $C_{2}$ respect to class $C_{1}$, for a given size $M$ of the sample, is the matrix $\Psi^{M}\left(C_{2} / C_{1}\right)$, expressed by

$$
\Psi^{M}\left(C_{2} / C_{1}\right)=\left\|\varsigma_{w, g} \Psi_{w, g}^{M}\left(C_{2} / C_{1}\right)\right\|
$$

where, $\Psi_{w, g}^{M}\left(C_{2} / C_{1}\right)=\left\lfloor m_{1}^{C_{2}}(w, g) / m_{1}^{C_{1}}(w, g), \cdots, m_{Q(w, g)}^{C_{2}}(w, g) / m_{Q(w, g)}^{C_{1}}(w, g)\right\rfloor$

is the sample contrast ratio, for parameter $w$ and statistics $g$.

The term, $\quad \psi_{q}^{M}\left(C_{2} / C_{1}, w, g\right)=m_{q}^{C_{2}}(w, g) / m_{q}^{C_{1}}(w, g), q=1, \ldots, Q$

is the specific sample contrast ratio, corresponding to moment $q$.

When the sample size is large enough, sample contrast ratios may be considered practically, population contrast ratios and by definition

$$
\Psi\left(C_{2} / C_{1}\right)=\lim _{M \rightarrow \infty} \Psi^{M}\left(C_{2} / C_{1}\right) \text { and } \Psi_{w, g}\left(C_{2} / C_{1}\right)=\lim _{M \rightarrow \infty} \Psi_{w, g}^{M}\left(C_{2} / C_{1}\right) .
$$

Suppose $M$ is large enough, the distance $d_{1,2}$ between classes $C_{1}$ and $C_{2}$ is given by

$$
d_{1,2}=\left\{\sum_{g=1}^{G} \sum_{w=1}^{W} \sum_{q=1}^{Q(w, g)}\left[m_{q}^{C_{2}}(w, g)-m_{q}^{C_{1}}(w, g)\right]^{2}\right\}^{1 / 2} .
$$

Making use of (7), distance $d_{1,2}$ given in (9) may be expressed by

$$
d_{1,2}=\left\{\sum_{g=1}^{G} \sum_{w=1}^{W} \sum_{q=1}^{Q(w, g)}\left\{\left[m_{q}^{C_{1}}(w, g)\right]^{2}\left[\psi_{q}^{M}\left(C_{2} / C_{1}, w, g\right)-1\right]\right\}^{2}\right\}^{1 / 2} .
$$


In the particular case of detection of anomalies from the background, i.e., when only two classes are considered, contrast ratio between these two classes takes the special denomination of anomaly / background ratio.

\section{The Optimum Border of a Class: A Likelihood Ratio Classifier}

Detection and classification of a radar target is a statistical pattern recognition problem. The proposed optimum classifier will be a likelihood ratio one, based on the Neyman - Pearson criterion, to determine the border of the class and to make possible the optimum decision in the moment space in accordance to RADASCE method. After processing the samples of the selected signal parameters, $\boldsymbol{n}$ independent moments are obtained, forming an $\boldsymbol{n}$-dimensional random variable $\mu_{i}$

$$
\mu_{i}=\left(m_{i 1}, \ldots, m_{i h}, \ldots, m_{i \Uparrow}\right), \quad i=1, \ldots, N, h=1, \ldots, n .
$$

This process is repeated $N$ times, resulting in the random variable $\mu$

$$
\mu=\left(\mu_{1}, \ldots, \mu_{i}, \ldots, \mu_{N}\right)
$$

When $M$ is large enough, theoretically when $M \rightarrow \infty$, the considered random variable, the moment $m_{i h}$ in our case, follows a normal distribution [ 7 ], given by

$$
p_{C}\left(m_{i h}\right)=\frac{1}{\sqrt{2 \pi} \sigma_{C h}} \exp \left[-\frac{\left(m_{i h}-m_{C h}^{P}\right)^{2}}{2 \sigma_{C h}^{2}}\right] \text {. }
$$

where, $C$ represents the class being analyzed. Two classes will be considered: $C=F, A$, where $F$ represents the background and $A$ an specific anomaly. The likelihood ratio $\Lambda(\mu)$ is expressed in this case, see complete analysis and demonstration in [ 5 ], by

$$
\Lambda(\mu)=\frac{p_{A}(\mu)}{p_{F}(\mu)}=\frac{\prod_{i=1}^{N} p_{A}\left(\mu_{i}\right)}{\prod_{i=1}^{N} p_{F}\left(\mu_{i}\right)} .
$$

where

$$
p_{A}\left(\mu_{i}\right)=\prod_{h=1}^{n} p_{A}\left(m_{i h}\right), \quad p_{F}\left(\mu_{i}\right)=\prod_{h=1}^{n} p_{F}\left(m_{i h}\right) .
$$

Taking into account (13) and (15), result the following expressions

$$
\Lambda(\mu)=\left[\prod_{h=1}^{n}\left(\frac{\sigma_{F h}}{\sigma_{A h}}\right)^{N}\right] \exp Z
$$




$$
\begin{gathered}
Z=\sum_{h=1}^{n} \sum_{i=1}^{N} Z_{h i} . \\
Z_{h i}=a_{h} m_{h i}^{2}+b_{h} m_{h i}+c_{h}
\end{gathered}
$$

where

$$
a_{h}=\left(\frac{\sigma_{A h}^{2}-\sigma_{F h}^{2}}{2 \sigma_{A h}^{2} \sigma_{F h}^{2}}\right), \quad b_{h}=\left(\frac{\sigma_{F h}^{2} m_{A h}^{P}-\sigma_{A h}^{2} m_{F h}^{P}}{\sigma_{A h}^{2} \sigma_{F h}^{2}}\right), \quad c_{h}=\left(\frac{\left(m_{F h}^{P}\right)^{2}}{2 \sigma_{F h}^{2}}-\frac{\left(m_{A h}^{P}\right)^{2}}{2 \sigma_{A h}^{2}}\right)
$$

The likelihood ratio $\Lambda(\mu)$ increases monotonously with $Z$ and it follows that, $\Lambda(\mu)=\Lambda_{0} \Rightarrow Z=Z_{0}$ and $\Lambda \geq \Lambda_{0} \Rightarrow Z \geq Z_{0}$. Therefore, $Z$ may be use as the decision statistics when Bayes criterion is applied.

Equation (17) represents a second order surface, whose specific form is determined by the signs of the invariants [8], obtained from $Z_{h i}, a_{h}, b_{h}, c_{h}$. For example, let be $n=3$ and $N=1$, and let's suppose (17) is the equation of an ellipsoid, represented in Fig. 2. This consideration does not implies loose of generality, but simplifies the explanation. For $Z=Z_{0}$, the axes $2 l_{1}, 2 l_{2}, 2 l_{3}$ and coordinates $\left(m_{10}, m_{20}, m_{30}\right)$ of the center of the ellipsoid are given by

$$
\begin{aligned}
& l_{1}=\left(\frac{Z_{0}}{a_{1}}\right)^{\frac{1}{2}}, \quad l_{2}=\left(\frac{Z_{0}}{a_{2}}\right)^{\frac{1}{2}}, \quad l_{3}=\left(\frac{Z_{0}}{a_{3}}\right)^{\frac{1}{2}} \\
& m_{10}=-\frac{b_{1}}{2 a_{1}}, \quad m_{20}=-\frac{b_{2}}{2 a_{2}}, \quad m_{30}=-\frac{b_{3}}{2 a_{3}}
\end{aligned}
$$

In radar target detection, Neyman - Pearson criterion is the most widely applied. In this case the probability of false alarm $P_{F}$ is fixed and the probability of detection is maximized. As it is known [9], Neyman - Pearson criterion is a particular case of Bayes criterion, in which, for a given $P_{F}$, corresponds a determined value of the threshold $\Lambda_{0}$ and, in our case, a second order surface (ellipsoid in the example considered) with determined parameters. For a constant false alarm probability $P_{F}$, the values of these parameters, and therefore the border of the class $F$ (the ellipsoid), may be found solving the equation

$$
P_{F}=1-\iiint_{W} p_{F}\left(m_{1}, m_{2}, m_{3}\right) d m_{1} d m_{2} d m_{3} \text {. }
$$

where, $p_{F}\left(m_{1}, m_{2}, m_{3}\right)$ is the joint probability density function of moments $m_{1}, m_{2}$, $m_{3}$, and $W$ is the integration region corresponding, in this case, to an ellipsoid. 


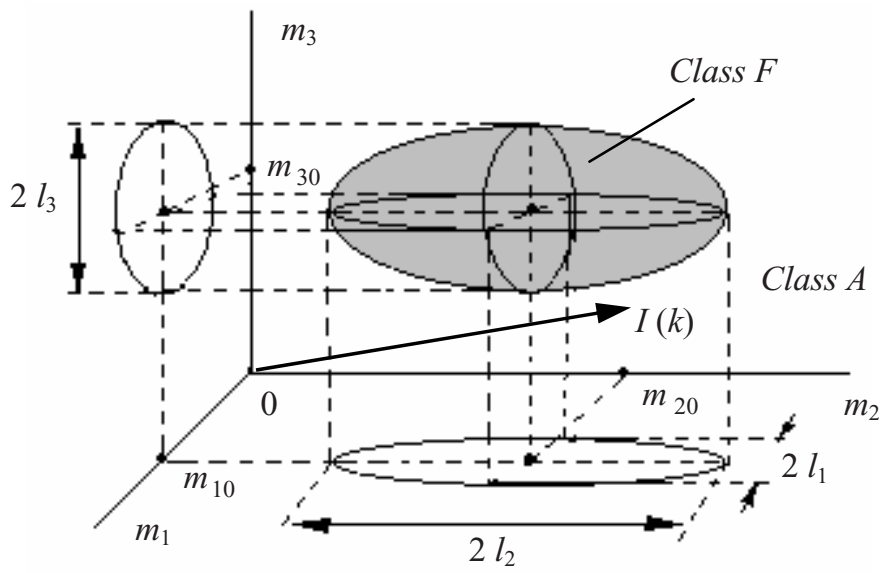

Fig. 2. Classification space and border of class $F$ (ellipsoid), for the case of three moments

\section{Classification Effectiveness of RADASCE}

There are only two possible classes to which the analyzed process may be assigned: Class $F$ and class $A$. The probability $P_{E}$ of an erroneous classification $[4,5]$ is given by

$$
P_{E}=P(F) P_{F}+P(A)\left[1-P_{D}\right] .
$$

For a given value of the false alarm probability $P_{F}$, increasing of the sample size $M$ will result in the increasing of the probability of detection $P_{D}$ [2 ], and, according to (22), the probability $P_{E}$ will decrease. The role of $M$ is shown in Fig. 3, where are presented the projections of the detection surfaces $\quad P_{D}$ vs $\left[\Psi_{q=1}^{M}(A / F, w, g), \Psi_{q=2}^{M}(A / F, w, g)\right] \quad[2] \quad$ on the plane
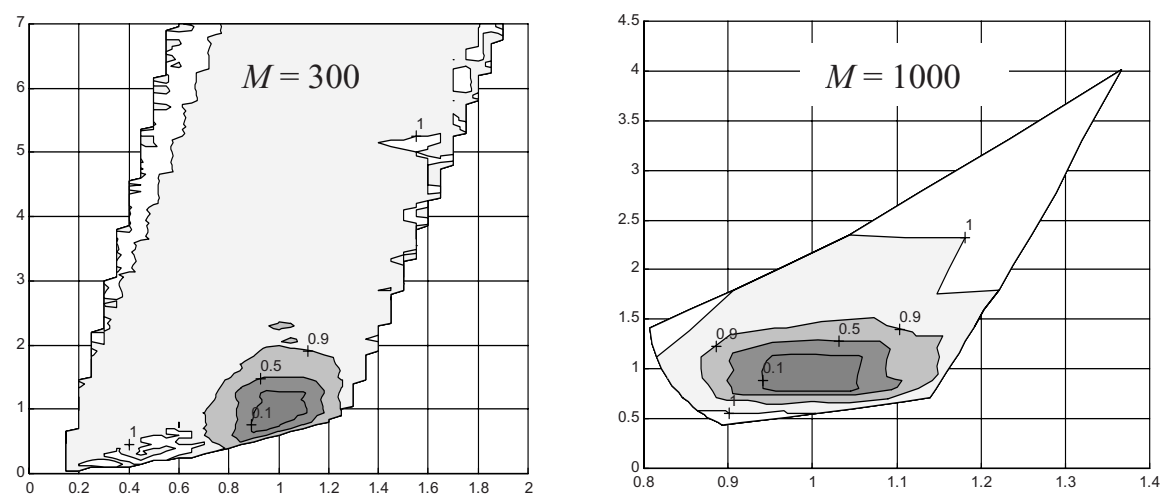

Fig. 3. Projections of two detection surfaces, for $M=300$ (left) and $M=1000$ (right), on the plane $\Psi_{q_{1}}^{M}(A / F, w, g), \Psi_{q_{2}}^{M}\left(A / F_{1}, w, g\right)$ 
$\Psi_{q=1}^{M}(A / F, w, g), \Psi_{q=2}^{M}(A / F, w, g)$, for: $\quad w=1$ (amplitude), $g=1, \quad Q(1,1)=2$, $q=1$ (first order moment) and $q=2$ (second order central moment).

Graphics in Fig. 3 were obtained using computer simulation in accordance to algorithms given in [ 2 ]; MATLAB was used for programming. The parameter $w$ is assumed to be Rayleigh distributed in the case of class $F$, and Rice distributed in the case of class $A$. From the analysis of these graphics follows that for equal values of contrast ratios, probability $P_{D}$ exhibits an appreciable increment when the sample size varies from $M=300$ to $M=1000$. The greater the number $\boldsymbol{n}$ of adequately selected moments, the better will be the quality of detection (recognition) of radar targets.

\section{Conclusions}

The use of a vector for decision in the moment space, whose components are a number of selected moments of signal parameters, obtained from large size samples, and the introduction of the concept of contrast ratio between classes ( in particular, the anomaly to background ratio instead of the classical signal to noise ratio), makes it possible to pass from a one dimension approach of the radar detection and recognition problem to a vector or multiple dimension one, more effective and with grater possibilities of radar target recognition. Two significant advantages of RADASCE are its intrinsic parametric nature and the possibility of controlling the detection probability through the sample size of the selected signal parameters. Experimental results have confirmed the high detection power of RADASCE.

\section{References}

1. Chávez, N., Chávez, L.V., Ravelo, A.: Radar Detection by Statistical Classification of the Cellular Emission of the Explored Region: Increasing of Detection employing Large Samples. ITM “José Martî” Science and Technology Review 2(1) (1999)

2. Chávez, N.: Radar Detection and Range: Early Warning for Targets in Masking Enviroments. A Solution to the Problem. Doctoral Dissertation. ITM "José Martî". Havana (2002)

3. Chávez, N.: Radar Detection and Recognition through the Analysis and Statistical Classification of the Cellular Emission. XIII Scientific Convention of Engineering and Architecture. ISPJAE - CUJAE, Havana, November - December (2006)

4. Chávez, N.: The Use of Large Size Samples and the Decision Making in the Moments Space: A New Approach to the Solution of the Radar Detection and Recognition Problem. Cuban Military Review of Science and Technology 1 (2007)

5. Chávez, N., González, A.L.: Optimization of Radar Detection when Decision Making Takes Place in the Moments Space. An Approach to the Solution of the Problem. Cuban Military Review of Science and Technology 3 (2008)

6. Lievin, B.R.: Theoretical Fundamentals of Statistical Radio Technique (in Russian). Radio and Communication, Moscow (1989)

7. Cramer, H.: Mathematical Methods of Statistics. Aguilar, Madrid (1970)

8. Korn, G.A., Korn, M.S.: Mathematical Handbook for Scientists and Engineers (in Russian). Science, Moscow (1977)

9. Helstrom, C.W.: Statistical Theory of Signal Detection. Pergamon Press, Oxford (1968) 